\title{
DUPLICATED RNA GENES IN TELEOST FISH GENOMES
}

\author{
Dominic Rose, Julian Jöris, Jörg Hackermüller, Kristin Reiche*, Qiang LI† Peter F. Stadler ${ }^{\ddagger}$ \\ Bioinformatics Group, Department of Computer Science, and Interdisciplinary Center for \\ Bioinformatics, University of Leipzig, Härtelstraße 16-18, D-04107 Leipzig, Germany \\ \{dominic,julian,kristin,stadler\}@bioinf.uni-leipzig.de, \\ \{joerg.hackermueller,kristin.reiche\}@izi.fraunhofer.de,q.li@fudan.edu.cn
}

\begin{abstract}
Teleost fishes share a duplication of their entire genomes. We report here on a computational survey of structured non-coding RNAs in teleost genomes, focussing on the fate of fish-specific duplicates. As in other metazoan groups we find evidence for a large number (11543) of structured RNAs, most of which $(\sim 86 \%)$ are clade-specific or evolve so fast that their tetrapod homologs cannot be detected. In surprising contrast to protein-coding genes, the fish-specific genome duplication did not lead to a large number of paralogous ncRNAs: only 188 candidates, mostly microRNAs, appear in a larger copy number in teleosts than in tetrapods suggesting that large-scale gene duplications do not play a major role in the expansion of the vertebrate ncRNA inventory.
\end{abstract}

Keywords: Non-coding RNA, ncRNA, ncRNA evolution, ncRNA gene finding, teleost fish, teleosts, Takifugu rubripes, whole-genome duplication, comparative genomics, annotation

\section{Introduction}

The heterogeneous class of non-protein-coding RNAs (ncRNAs) has in recent years moved from a biochemical curiosity to a main research topic in Molecular Biology. Recent results from high-throughput transcriptomics ${ }^{1,2,3,4}$ have established that ncRNAs in fact dominate the transcriptomes of higher eukaryotes, even though the list of non-protein coding RNAs is still largely incomplete. With ncRNAs being implicated in a plethora of regulatory $\operatorname{roles}^{5,6}$, it becomes an interesting issue to understand their evolution in more detail and beyond the mostly anecdotal narratives available for individual ncRNA gene families, $\mathrm{see}^{7}$ for a review.

Compared to the annotation of protein-coding genes, non-coding RNA annotation of genomic sequences is still in its infancy. This is true in particular beyond

*Primary Affiliation: Fraunhofer Institute for Cell Therapy and Immunology, Perlickstr. 1, 04103 Leipzig, Germany

${ }^{\dagger}$ Primary Affiliation: T-Life Research Center, Fudan University, Shanghai 200433, China

¥Secondary Affiliations: Fraunhofer Institute for Cell Therapy and Immunology, Perlickstr. 1, 04103 Leipzig, Germany; Department of Theoretical Chemistry, University of Vienna, Währingerstraße 17, A-1090 Wien, Austria; Santa Fe Institute, 1399 Hyde Park Rd., Santa Fe, NM 87501, USA 
(A)

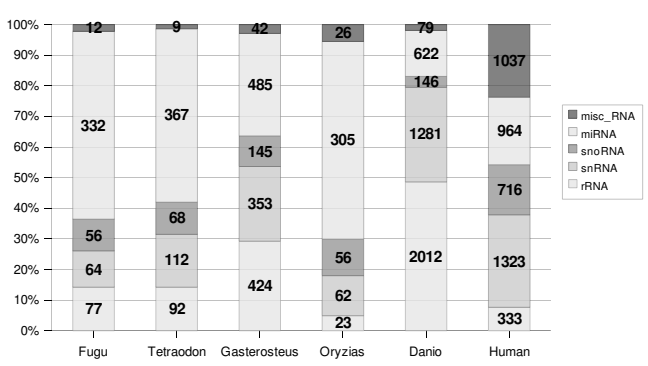

(B)

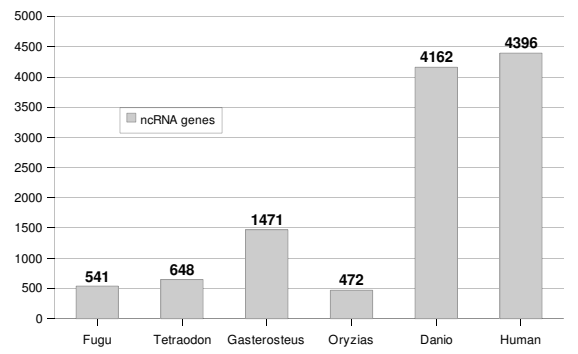

Fig. 1. Ensembl-48 annotation of human and teleost fish genomes.

mammalian genomes, which mostly "inherit" the human and mouse annotation in a straightforward way. A particular problem for all large-scale studies of ncRNA evolution are massive differences in ncRNA coverage and the biases in annotation even between fairly closely related organisms. In addition to biased coverage, the currently available ncRNA annotation procedures cannot distinguish clearly between functional ncRNAs and the sometimes huge number of associated pseudogenes. Fig. 1 summarizes the situation for the five available teleost fish genomes, making it obvious that a systematic study of ncRNA evolution cannot be meaningfully performed solely on the basis of the available annotation.

A further drawback of current annotation procedures it that they typically consider only a small subset of the ncRNA universe. Beyond the "house keeping" RNAs (tRNA, snRNAs, RNAse P and MRP RNA and a few Pol III transcripts such as vault and Y RNAs), annotation is mostly restricted to microRNAs and the two classes of snoRNAs. In contrast, computational studies have provided convincing evidence for tens of thousands of RNAs whose secondary structure is under stabilizing selection ${ }^{1,8,9,10}$, while even more functional ncRNAs without evolutionarily conserved structure were found in the large-scale transcriptomics projects mentioned above.

Here, we study the overall characteristics of ncRNA evolution in teleost genomes. From an evolutionary perspective, teleost fish genomes are of particular interest because they have undergone a complete duplication of their genomes - usually called the Fish Specific Genome Duplication (FSGD) - just before the radiation of the crown-group teleosts ${ }^{11,12}$. This raises in particular the question to what extent duplicated ncRNAs are retained after such a large-scale duplication event.

Thus, the purpose of this contribution is two-fold. First we report an RNAzbased survey of teleost fish genomes. We demonstrate that like many other groups of organisms (mammals ${ }^{8,9}$, urochordates $^{13}$, nematodes ${ }^{14}$, insects ${ }^{15}$, and yeasts ${ }^{16}$ ) teleost genomes contain a large number of previously undescribed, clade-specific, non-coding RNAs. Secondly, we investigate from a global perspective the fate of 


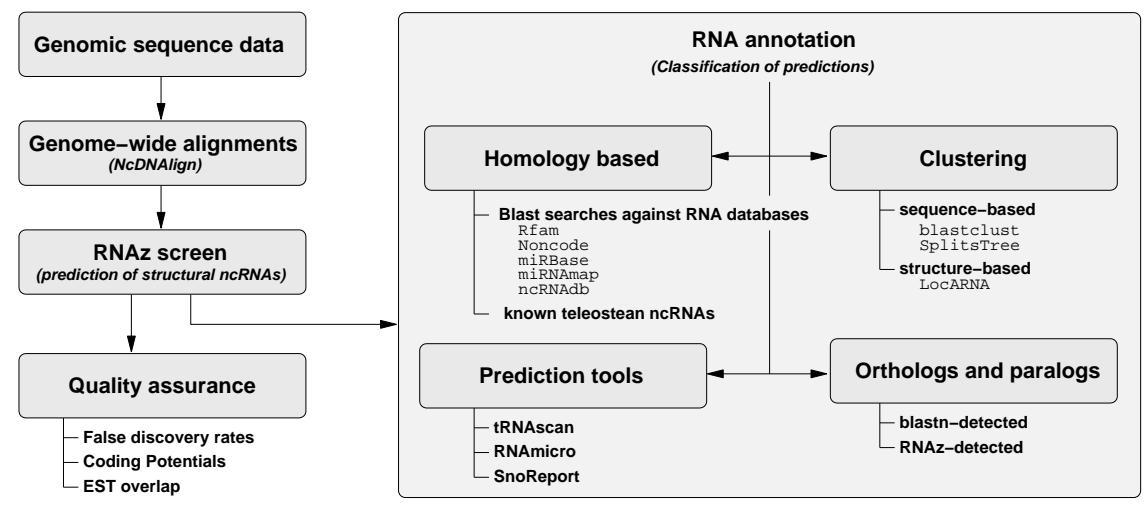

Fig. 2. Overview of the computational workflow.

ncRNAs in the wake of a genome duplication.

\section{RNAz Screen: Unbiased Prediction of Structured RNAs}

We prepared genome-wide alignments of the non-repetitive non-protein-coding DNA of the five available teleost genomes (Ensembl-48) (fugu,Takifugu rubripes, Tr; pufferfish, Tetraodon nigroviridis, Tn; stickleback, Gasterosteus aculeatus, Ga; medaka, Oryzias latipes, Ol; and zebrafish, Danio rerio, Dr) using NcDNAlign ${ }^{17}$ with fugu serving as the reference organism. Due to the larger evolutionary distances among teleosts compared to mammals, only a relatively small fraction of the genomes can be reliably aligned. In order to improve the performance, we consider only DNA that is alignable to the fugu genome after removing all "known coding sequences". Local alignments containing at least three species were then scored using the RNAz package $(\mathrm{v} 1.0)^{18}$. RNAz is a machine learning approach that allows the prediction of structural non-coding RNAs. It classifies given alignment slices as either "ncRNA" or "other" by analysing secondary structure conservation and thermodynamic stability of the fold. Alignments are scanned by moving a $120 \mathrm{nt}$ window with a step-width of $40 \mathrm{nt}$, so that consecutive slices overlap in $80 \mathrm{nt}$. This sliding-window mechanism is motivated by the fact that many structured RNAs are shorter than $100 \mathrm{nt}$. Such small RNA signals would "drown" in the noise of longer, mostly unstructured, alignments. On the other hand, there is no reliable signal for secondary structure conservation in too short alignments. Previous RNAz-based studies discuss these issues in more detail ${ }^{8,15,16}$. A brief overview of our computational procedure is presented in Fig. 2.

At RNAz classification probabilities of $p>0.5$ and $p>0.9$ we obtained 19916 and 6690 "structured" sequence windows, which can be combined into 11543 and 4407 predicted structured RNA elements, respectively. In order to estimate the false discovery rate (FDR), the entire screen has been repeated using column-wise shuffled alignments as input. As described in $^{19}$ and tested in practice in ${ }^{8,9,13,14,15}$, 
Table 1. Summary of the RNAz screen.

\begin{tabular}{|l|l|llll|}
\hline Species & Tr & Tn & Ga & Ol & Dr \\
\hline genome size $[\mathrm{Mb}]$ & 393 & 402 & 462 & 868 & 1440 \\
without CDS [Mb] & 361 & 211 & 429 & 838 & 1410 \\
non-coding alignments & 66448 & 30369 & 56750 & 49958 & 9027 \\
aligned DNA [Mb] & 8.45 & 4.9 & 8.8 & 5.44 & 0.96 \\
\hline scored by RNAz [Mb] & 10.7 & 5.84 & 5.41 & 7.7 & 1.3 \\
\hline RNAz p $>0.5$ & 11543 & 5480 & 9897 & 8970 & 1623 \\
$\quad[\mathrm{~Kb}]$ & 1469 & 692 & 1229 & 1116 & 201 \\
RNAz p $>0.9$ & 4407 & 2186 & 3693 & 3412 & 723 \\
$\quad[\mathrm{~Kb}]$ & 602 & 260 & 431 & 398 & 85 \\
\hline FDR p $>0.5[\%]$ & 26 & 26 & 26 & 26 & 26 \\
FDR p $>0.9[\%]$ & 18 & 18 & 19 & 18 & 16 \\
\hline
\end{tabular}

the shuffling procedure of the RNAz-package generates randomized alignments that preserves the salient characteristics of multiple alignments: length, base composition, gap patterns, and conservation patterns. Comparing the amount of positively scored DNA from control and normal screen yields FDR estimates of $26 \%$ and $18 \%$ for the low and high confidence levels. Alternatively, we can base FDR estimates on the numbers of positively scored sequence windows and observe similar FDRs: $26 \%(5131 / 19916, p>0.5)$ and $18 \%(1207 / 6690, p>0.9)$. These estimates might be somewhat optimistic since the shuffling algorithm of the RNAz-package does not account for dinucleotide content. Preserving dinucleotide content while generating randomized alignments is essential to accurately assess the significance of the prediction ${ }^{20}$ and previous RNAz-based studies have shown that taking this effect into account substantially increases the estimated FDR on mammalian sequences (FDR of $\sim 50 \%$ for the ENCODE regions ${ }^{9}$ ). In contrast, the impact of using dinucleotide instead of mononucleotide shuffling has been quite small on drosophilid sequences 15. As an additional control, we therefore applied SISSIz ${ }^{21}$, a novel approach to generate dinucleotide-controlled random alignments with the characteristics of a given input alignment. Not unexpectedly, the estimated FDR indeed increased to up to $68 \%$. The SISSIz-randomized control screen still contained 41 known ncRNAs, indicating that SISSIz leads to a conservative (pessimistic) FDR estimate. Tab. 1 summarizes the statistics of the RNAz screen.

We excluded currently annotated protein-coding sequences (Ensembl-48) from our analysis. The coding potential scores of $\mathrm{CPC}^{22}$ further suggest that almost all RNAz hits are indeed in non-coding regions: only $100(<1 \%)$ are predicted as "coding", a value that is less than CPC's false positive rate, which its authors estimate at $\sim 2 \%$.

In order to obtain at least a rough estimate of the sensitivity, we compared the 
Table 2. Sensitivity of RNAz on Ensembl-48-annotated fugu ncRNAs. Denoted percentages refer to the number of recovered ncRNAs using RNAz $(p>0.5)$ over the number of ncRNAs present in the input alignments.

\begin{tabular}{|lrrrr|}
\hline class & RNAz & input & Ensembl-48 & sensitivity (\%) \\
\hline rRNAs & 66 & 77 & 77 & 86 \\
snRNAs & 49 & 64 & 64 & 77 \\
snoRNAs & 22 & 55 & 56 & 40 \\
miRNAs & 261 & 276 & 332 & 95 \\
other & 11 & 11 & 12 & 100 \\
\hline all & 409 & 483 & 541 & 85 \\
\hline
\end{tabular}

RNAz output with existing annotation. Since annotated repetitive elements as defined by RepeatMasker have been removed from our input data, several classical RNA families have also been excluded, in particular tRNAs, some of the snRNAs and most of the "misc_RNAs". Only 321 of 541 known fugu ncRNAs passed the repeat masking step and only 245 of these are sufficiently well conserved to be alignable with homologous sequences of the remaining teleosts. Of these, $221 \mathrm{ncR}-$ NAs $(90 \%)$ are recognized by RNAz. In order to obtain a more realistic sensitivity estimate, we retrieved all annotated ncRNAs from the fugu genome, added $100 \mathrm{nt}$ of flanking sequence on both sides, and used NcDNAlign to retrieve their homologs and to construct multiple alignments. These were then scored with RNAz using the same parameters as the main screen. We obtained an overall sensitivity of about $85 \%$, see Tab. 2. As in previous work, we note that in particular for snoRNAs the sensitivity is poor, while sensitivity values for miRNAs are encouraging. A considerable number of predicted ncRNAs overlap ESTs and hence show evidence for active transcription, cp. Table 3 .

\section{RNA Annotation}

Our annotation procedure follows a recently published scheme ${ }^{23}$. Overall, 1372 RNAz hits can be annotated by the following protocol: Firstly, known RNA genes and homologs of known ncRNAs were identified based on sequence comparison, here using blastn searches against all major ncRNA databases: Rfam $8.0^{24}$, NONCODE $2.0^{25}$, miRBase $10.0^{26}$, miRNAmap $1.0^{27}$, ncRNAdb ${ }^{28}$ (number of RNAz hits showing sequence conservation with entries of respective ncRNA database: 115 Rfam, 104 NONCODE, 208 miRBase, 179 miRNAmap, 71 ncRNAdb). In the second step, we used specialized programs to recognize novel members of three ncRNA classes. Since tRNAs were removed from the input set as multi-copy genes, we found only one tRNA and two tRNA pseudogenes with tRNAscan ${ }^{29}$. An experimental version of SnoReport ${ }^{30}$ identified 885 snoRNAs (727 CD- and 136 HACA-Box snoRNAs, 22 are classified as both), of which 8 match previously annotated miRNAs. This is 
Table 3. A comparison of ncRNA candidates and EST sequences provides evidence for transcriptional activity. The table lists the number of fugu loci obtained by BLAST searches (E-value < $1 \mathrm{e}-5)$ between fugu sequences from (1) our teleostean ncRNA candidates $(p>0.5$, overall 11543 loci) and (2) the tetrapod-conserved subset (overall 1581 loci, cp. Tab. 4) against EST sequences of four teleosts provided by the UCSC Table Browser. The small number of matches with fugu ESTs may be due to the fact that UCSC only offers access to sequences of a deprecated fugu assembly which is only partially compatible with the Ensembl data our study is based on. For tetraodon, no ESTs were available. The last column lists the hits to the medaka EST-database (http://medaka.lab.nig.ac.jp/est_index.html).

\begin{tabular}{|lrrrr|r|}
\hline ESTs of & $\mathrm{Tr}$ & $\mathrm{Ga}$ & $\mathrm{Dr}$ & $\mathrm{Ol}$ & m-EST-DB \\
\hline$(1)$ & 796 & 3847 & 2080 & 3558 & 644 \\
annotated & 292 & 1436 & 768 & 2229 & 63 \\
unknown & 504 & 2411 & 1314 & 1329 & 581 \\
\hline$(2)$ & 192 & 616 & 844 & 693 & 173 \\
annotated & 33 & 159 & 194 & 159 & 42 \\
unknown & 159 & 457 & 650 & 534 & 131 \\
\hline
\end{tabular}

within the expected FDR of SnoReport. We used RNAmicro ${ }^{31}$ to determine putative miRNA precursors. At a confidence level of $p_{\text {RNAmicro }}>0.5$, we obtained 434 candidates, of which 190 have blastn matches with miRBase or miRNAmap entries. Fig. 3 summarizes the miRNA annotation in some more detail.

An example of a novel fugu miRNA candidate, not yet listed in Ensembl-48, is a homolog of xtr-miR-449, Fig. 4 (top). The structure shows all hallmarks of a microRNA precursor including the characteristic conservation pattern. Another novel miRNA precursor candidate found by RNAz and RNAmicro is the intronic locus2693 (scaffold_204, pos. 260585-260681), which contains the mature sequence of dre-miR-728. It is also not yet included in Ensembl-48's annotation tracks.

As a third step, we employed the structural clustering procedure proposed by Will et $a .^{32}$ to find possible novel structural classes. All RNAz hits with either a classification probability exceeding 0.9 or a valid annotation form the input of the clustering procedure (2 293 loci). In brief, a modified Sankoff algorithm is used to compute local structural alignments and their consensus structure. The cluster-tree (see Fig. 6) is then obtained by agglomerative clustering based on the alignment scores. It is further processed to identify an optimal partition by evaluating the squared error of the minimum free energies of the individual sequences located within the subtree rooted at an internal node relative to the minimum free energy of their common consensus secondary structure. If the increase of the squared error is unexpectedly high when traversing the cluster-tree from the leaves to the root node, the merging of two internal nodes to one common cluster is stopped. In this way, we estimate that there are at least 106 relevant clusters (which depend on several thresholds, including the chosen cutoff to stop the merging procedure, a structure conservation index $(\mathrm{SCI})>0.7$, and a consensus minimum free energy $(\mathrm{MFE})<$ 


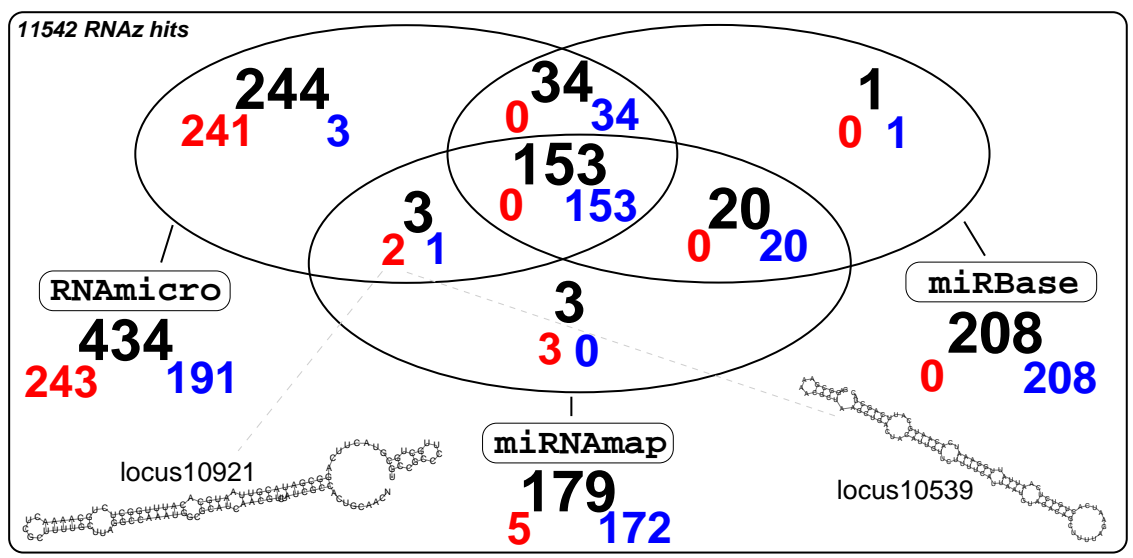

Fig. 3. MicroRNA annotation of RNAz hits. The Venn diagram contrasts the number of RNAz predictions that are positively classified by RNAmicro and hits that have also a miRBase and/or a miRNAmap BLAST hit. For each subset we record three numbers in the form $y x_{z}$ with $y+z=x$, where $x$ is the number of microRNA precursors in the intersection of all three approaches, $z$ is the number of corresponding miRNAs annotated in Ensembl, and $y$ is the number of miRNAs not annotated in Ensembl (these structures are the most interesting ones because they constitute putatively novel miRNAs).

$-20 \mathrm{kcal} / \mathrm{mol}$ ) having an average cardinality of 2.9 sequences, an average SCI of 0.89 , and an average MFE of $-34.97 \mathrm{kcal} / \mathrm{mol}$. Fig. 6 displays the resulting cluster tree and Fig. 4 (bottom) illustrates an example-subtree in more detail. Beside known ncRNAs, both trees contain novel, closely related structures demonstrating that our clustering approach indeed yields useful classifications. As in other recent work ${ }^{32,33}$, we predict several new miRNA candidates by means of clustering, including some candidates that are not recognized by RNAmicro.

Of particular interest are sets of unannotated RNAz hits that do not only fold into similar structures and hence are identifiable in the cluster tree, but which are also located in close vicinity on the genome. Such arrangements are observed e.g. for polycistronic microRNA transcripts ${ }^{34}$, for multiple unrelated snoRNAs sharing the same host gene ${ }^{35}$, and for several Pol III transcripts, including Y RNAs ${ }^{36,37}$ and vault RNAs. Altuvia et al. ${ }^{34}$ argued that miRNA precursors that are located within short chromosomal distances $(<3000 \mathrm{nt})$ from each other most likely arise from polycistronic transcripts. Interestingly, the 434 RNAmicro predictions contain 41 such clusters. However, there are only six such clusters within the 246 "novel" miRNA candidates, each comprising either two or three loci only. Overall, we observe 1223 genomic clusters with a maximal distance of $1 \mathrm{~kb}$ consisting of 2 to 8 loci. Increasing the cut-off distance to $10 \mathrm{~kb}$ yields 1912 genomic clusters, the largest comprising 110 sequences. Within the high confidence $p_{\text {RNAz }}>0.9$ predictions, we still find 234 clusters (730 at $10 \mathrm{~kb}$ ), each with up to 5 (20) loci. Fig. 5 shows the distribution of pairwise LocARNA distances used in structural clustering for RNAz 

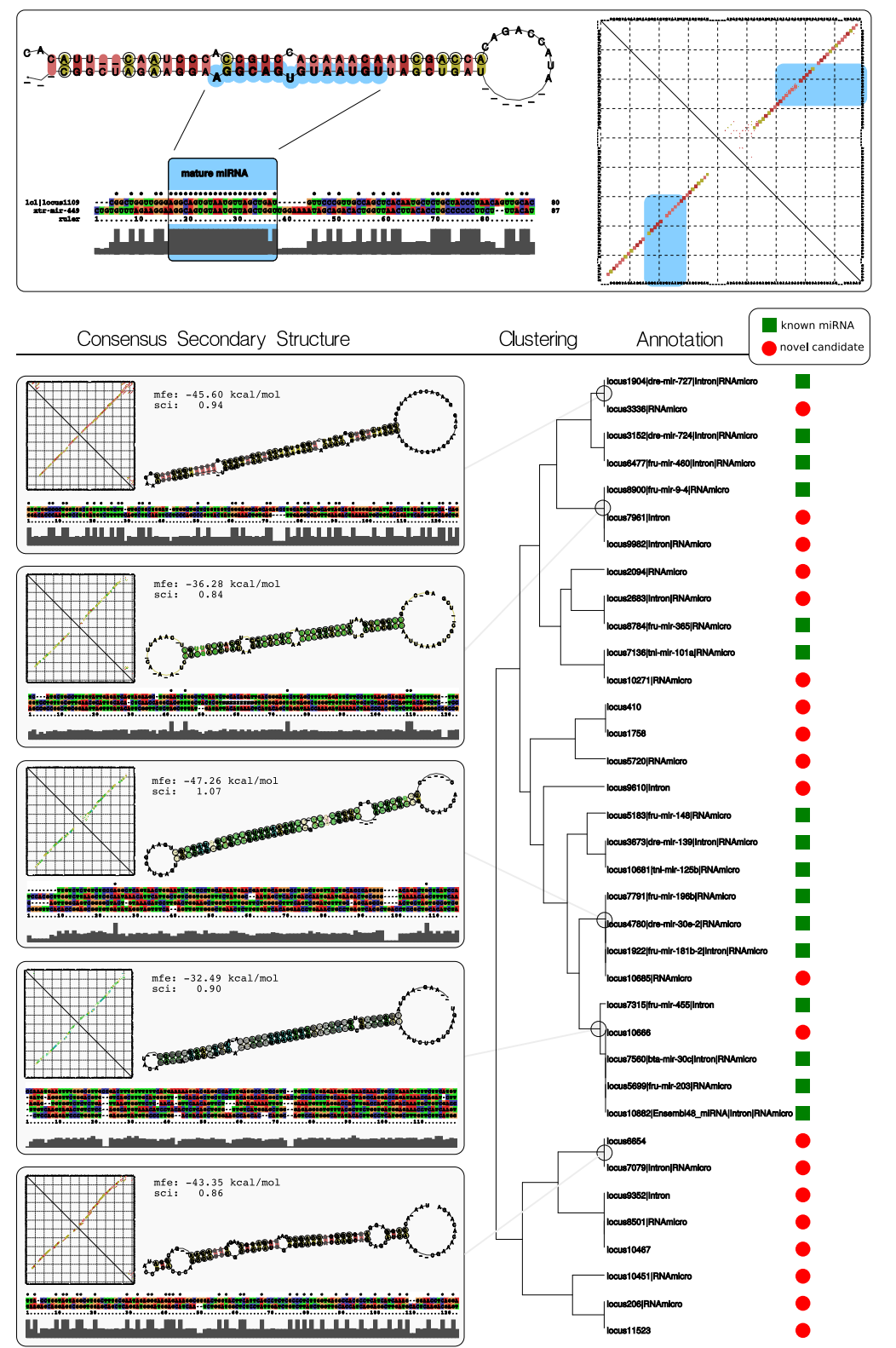

Fig. 4. Novel microRNAs in teleost genomes.

Top. Fugu miRNA-449 (locus 1285, scaffold_360, pos. 9131-9211) is an example of a homolog of a known miRNA not annotated in Ensembl. This RNAz prediction is classified as miRNA precursor by RNAmicro and a BLAST search against the mature miRNAs in miRBase reveals that it contains the sequence of the mature miRNA xtr-miR-449.

Below. Structure-based clustering reveals several collections of hairpin structures. These clusters contain already known miRNAs, but also closely related novel miRNA precursor candidates. Primary sequences of this example vary considerably, but secondary structure motives are significantly well conserved. Novel ncRNA candidates (red circle) with a positive RNAmicro classification are prime candidates for novel miRNAs. 


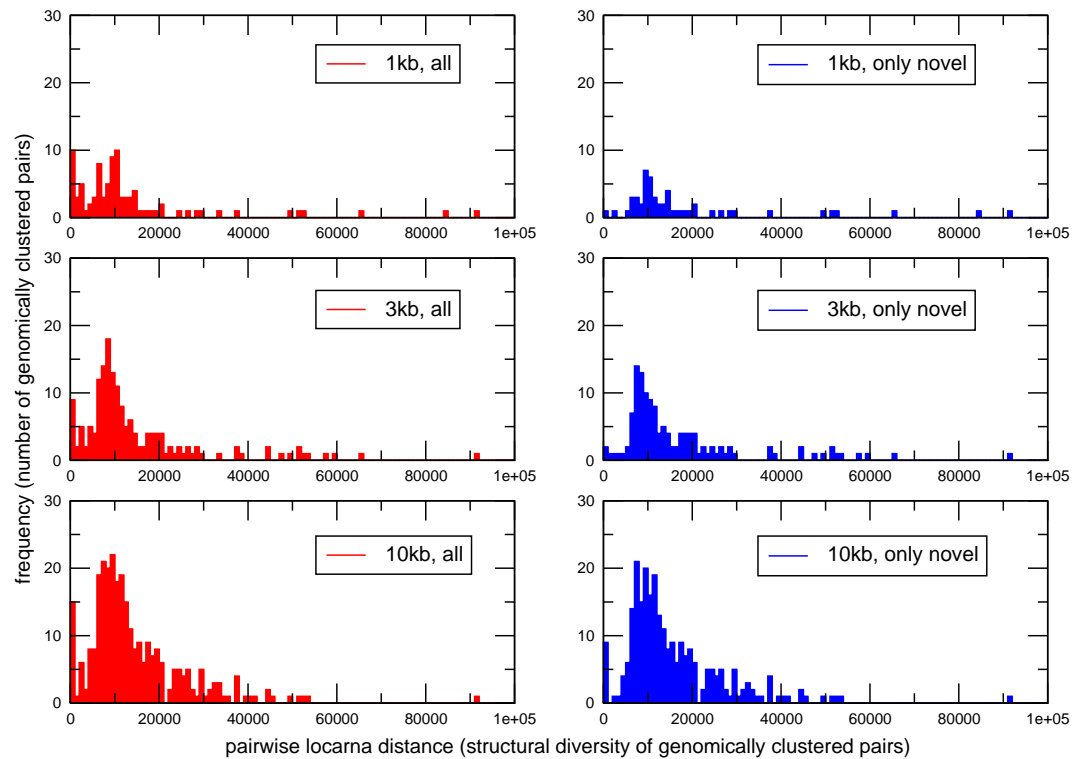

Fig. 5. Distribution of structure distances between pairs of adjacent high confidence $\left(p_{\text {RNAz }}>0.9\right)$ RNAz hits with a maximum distance of $1 \mathrm{~kb}, 3 \mathrm{~kb}$ and $10 \mathrm{~kb}$, respectively. The left column shows histograms for all genomic clusters of RNAz predictions, the right column is restricted to clusters containing putatively novel structures that are not included at Ensembl-48.

hits that form genomic clusters. The distribution is clearly bi-modal, consisting of a random bulk and a small set of signals with very similar structures. While fairly restrictive, the data indicate that there are at least a hand full of novel structural classes of RNAs that tend to cluster in the same genomic location.

\section{Orthologs and Paralogs}

A BLAST search $\left(\right.$ E-value $\left.<10^{-5}\right)$ shows that $944(8 \%)$ of the fugu RNAz predictions have sequence similarity with highly conserved non-coding vertebrate elements $(\mathrm{hCNE})^{38}$, of which 246 candidates have some annotation. 541 loci are not only alignable with vertebrates (human, mouse, dog, chicken, shark using NcDNAlign), the extended alignments are still classified as structured RNAs by RNAz, see Tab 4 . Within this set of highly conserved structures, the estimated FDR significantly reduces to only $7-11 \%$, and only two sequences are classified as coding by CPC. 164 of these sequences have homologs within the set of 997 high-confidence highly conserved predictions of the prior mammalian RNAz screen ${ }^{8}$. Our data thus provide at least 377 additional well-conserved ncRNA candidates that were not detected by 
the earlier survey.

According to the UCSC Table Browser, at least 250 teleostean ncRNA candidates $(2 \%)$ have sequence similarity with untranslated regions (UTRs) of human protein-coding genes (5'UTRs: 136, 3'UTRs: 162, some match both types of UTRs). Regarding the set of tetrapod-conserved RNAz predictions, 140 (26\%) out of 541 candidates cover human intronic sequences and 181 match human UTRs (5'UTRs: 112, 3'UTRs: 116). Due to the lack of a reliable fugu UTR annotation we analysed the $1 \mathrm{~kb}$ flanking region of the 5' and 3' boundaries of Ensembl's fugu protein-coding genes. Interestingly, the 5' flanking regions (3' flanking regions) contain 1505 (1448) RNAz hits of which $94 \%$ (93\%) are potential cis-regulatory signals of unknown function. Among the tetrapod-conserved RNAz hits, 114 candidates reside within $1 \mathrm{~kb}$ up or down-stream of protein-coding genes, 61 of which are not annotatable.

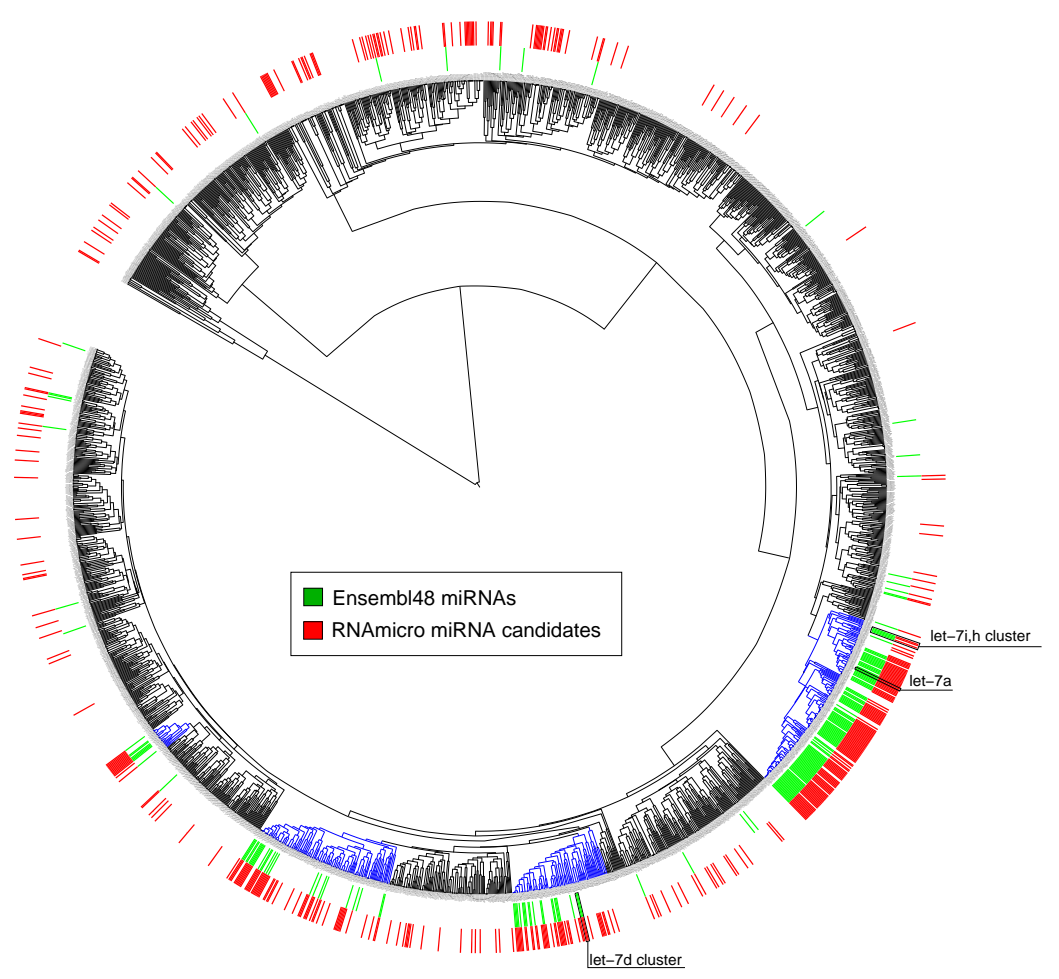

Fig. 6. Cluster-tree of chosen high-scoring teleostean ncRNA candidates.

The tree comprises 4585 nodes, of which 2293 are leaves. Known fugu miRNAs as provided by Ensembl-48 are indicated in green (inner circle), RNAmicro-predicted miRNA candidates are marked in red (outer circle). Certain subtrees containing typical miRNA clusters are drawn in blue. Encouragingly, the majority of known miRNA clusters is recovered (red+green). Overall, there is substantial evidence for novel miRNA(-like) classes of ncRNA structures in teleostean genomes (red only). 
Table 4. Phylogenetic conservation of fugu RNAz predictions. For all RNAz hits $\mathrm{p}>0.5$ we list the number of elements with blastn-detected homologs (left part) and the number of hits that again were classified as structured (RNAz) when aligned with the sequences from human, mouse, dog, chicken, or shark (right part). By considering both approaches, we overall obtain 1581 tetrapodconserved ncRNA candidates.

\begin{tabular}{|c|c|c|c|c|c|c|}
\hline \multirow[b]{2}{*}{ Genome } & \multicolumn{3}{|c|}{$\begin{array}{l}\text { blastn hit } \\
\text { annotation }\end{array}$} & \multicolumn{3}{|c|}{$\begin{array}{c}\text { positive RNAz predictions } \\
\text { annotation }\end{array}$} \\
\hline & all & yes $(\%)$ & no $(\%)$ & all & yes $(\%)$ & no $(\%)$ \\
\hline Any non-teleost & 1487 & $278(19)$ & $1209(81)$ & 541 & $230(43)$ & $311(57)$ \\
\hline Homo sapiens & 989 & $222(22)$ & $767(78)$ & 478 & $208(44)$ & $270(56)$ \\
\hline Gallus gallus & 1050 & $217(21)$ & $833(79)$ & 455 & $204(45)$ & $251(55)$ \\
\hline Canis familiaris & 1010 & $225(22)$ & $785(78)$ & 467 & $203(44)$ & $264(56)$ \\
\hline Mus musculus & 912 & $211(23)$ & $701(77)$ & 429 & $194(45)$ & $235(55)$ \\
\hline Callorhinchus milii & 610 & $153(25)$ & $457(75)$ & 357 & $190(53)$ & $167(47)$ \\
\hline Petromyzon marinus & 188 & $40(21)$ & $148(79)$ & - & - & - \\
\hline Drosophila melanogaster & 32 & $21(66)$ & $11(34)$ & - & - & - \\
\hline Caenorhabditis elegans & 8 & $5(63)$ & $3(37)$ & - & - & \\
\hline
\end{tabular}

In contrast, only a tiny fraction of our structured RNA candidates seems to be conserved within any of the available invertebrate genomes. Again, note that this observation is misleading because the best-conserved "house keeping" ncRNAs, in particular tRNAs and snRNAs, were removed from the input data.

From an evolutionary perspective, an important issue is the fate of duplicated non-coding RNA signals. Since the ancestral vertebrate genome already went through two rounds of whole-genome duplications, we have to expect up to eight paralogs in teleost genomes. For the case of microRNAs we demonstrated in previous work that duplicated ncRNAs frequently have survived the genome duplication $(\mathrm{s})^{39}$. We therefore compared the RNAz predictions at sequence level and list them by their copy number in Tab. 5. The repeat-masked RNAs are not informative for this purpose because their copy numbers are highly variable between relatively closely related species, see supplemental data.

The fraction of predictions with more than eight copies seems to contain repetitive or pseudo-genic elements rather than correctly identified, evolutionary duplicated ncRNAs. The fraction of unannotated hits grows with larger number of copies. One explanation for this observation might be that these sequences diverged by accumulating mutations after they got duplicated, but simultaneously preserve their secondary structure, so that it becomes more and more difficult to reliably annotate them comparatively at the sequence level, while they still remain detectable by algorithms that incorporate structural features, e.g. RNAz or LocARNA. Interestingly, the mutual distance obtained by structure-based clustering between duplicated ncRNA 
Table 5. Distribution of paralogous fugu RNAz hits. Paralogs are obtained by BLAST searches (E-value $<1 \mathrm{e}-3)$ of the fugu RNAz candidate sequences against themselves. They are compared with each other to exclude likely pseudogenes. We observe 1403 duplicated loci, 964 of which have two to eight copies. Furthermore, we provide the number of RNAz hits conserved between fugu and human, which occur more often in fugu than in human (and the other way round). As an example, 135 ncRNA candidates, present as single copy in human, occur more than once in fugu. Conversely, 81 candidates, not duplicated in fugu, appear multi-copied in human.

\begin{tabular}{|l|r|rrrrrrr|r|}
\hline \# copies & 1 & 2 & 3 & 4 & 5 & 6 & 7 & 8 & $>8$ \\
\hline p $>0.5$ & 10139 & 573 & 150 & 82 & 50 & 39 & 36 & 34 & 439 \\
annotated & 379 & 85 & 45 & 32 & 25 & 8 & 8 & 4 & 51 \\
unknown & 9760 & 488 & 105 & 50 & 25 & 31 & 28 & 30 & 388 \\
teleost-specific & 8964 & 409 & 93 & 48 & 23 & 30 & 19 & 30 & 345 \\
in tetrapods & 1175 & 164 & 57 & 34 & 27 & 9 & 17 & 4 & 94 \\
in human & 777 & 103 & 35 & 25 & 23 & 8 & 7 & 4 & 7 \\
fugu > human & 135 & 34 & 18 & 1 & 0 & 0 & 0 & 0 & 0 \\
human > fugu & 81 & 7 & 0 & 0 & 0 & 0 & 1 & 0 & 0 \\
\hline p $>0.9$ & 3689 & 253 & 67 & 75 & 44 & 25 & 25 & 18 & 211 \\
annotated & 238 & 68 & 37 & 34 & 27 & 6 & 0 & 2 & 46 \\
unknown & 3451 & 185 & 30 & 41 & 17 & 19 & 25 & 16 & 165 \\
teleost-specific & 3180 & 162 & 26 & 38 & 12 & 18 & 14 & 16 & 171 \\
in tetrapods & 509 & 91 & 41 & 37 & 32 & 7 & 11 & 2 & 40 \\
fugu > human & 94 & 28 & 17 & 1 & 0 & 0 & 0 & 0 & 0 \\
human > fugu & 38 & 1 & 0 & 0 & 0 & 0 & 0 & 0 & 0 \\
\hline
\end{tabular}

candidates increases with the number of loci that belong to the cluster (see supplement). This could be explained by a duplication/deletion mechanism in which cluster members are destroyed at random by mutation as the exact copy number is not under strong selection.

Comparing the number of ncRNAs that occur at most eight times in fugu with the copy number in human (cp. Tab. 5) reveals that in 188 cases teleosts contain more ncRNA copies than tetrapods, of which 74 are of unknown function. In turn, tetrapods contain the higher number of copies in only 89 cases. These data indicate that, with the most notable exception of microRNAs ${ }^{39}$, additional copies of non-coding RNAs are rarely retained in the aftermath of the fish-specific genome duplication. The loss appears to be more extensive than for protein-coding genes, where at least about 1000 paralogs arising from the fish specific genome duplication have been reported in the genomes of fugu and tetraodon ${ }^{40}$. It is conceivable, however, that duplicated ncRNAs have diverged so far that they are not recognized as paralogs by blast-based methods.

To illustrate the fate of ncRNAs subsequent to duplication in more detail, we estimated the densities of the bivariate distribution of sequence versus structural similarity over duplicated and randomly chosen pairs of sequences (Fig. 7). Duplicated loci can be assigned to three distinct groups (Fig. 7 C): those, where both sequence and structure are nearly identical (p1); a set with significant sequence di- 
vergence but negligible structural differences (p2), and a set which largely overlaps with the background distribution of randomly chosen pairs (p3). The ostensible discrepancy between the E-value of the initial BLAST search to identify duplicated pairs and the mean pairwise identity of the ClustalW alignment used here is a consequence of scoring differences between local and global alignments: subsequences of both loci are maintained with high similarity (which is what blast focuses on), whereas overall the loci may be quite highly divergent, both in terms of sequence and structure. This might be interpreted as a process that maintains some local functional features, presumably those that are required to determine an RNA type, e.g. a protein binding site for a guide RNA, whereas the functional role can diverge quickly upon duplication. A related behavior can be observed for duplicated miRNAs, which mainly make up for the p2 peak (cp. Suppl. Fig. 4). Upon duplication, miRNAs largely maintain their precursor structure, but diverge on the sequence level - the affiliation to a particular functional class is maintained - whereas the functional role, the target specificity, changes. As expected, duplicated snoRNAs do not share such strict constraints, they are found at any hotspot position of the density plots. Similarly, the non-annotatable loci appear also widely spread throughout the landscape. No other annotated classes of ncRNAs are found in the set of duplicated pairs.

The small number of ncRNA candidates that have two paralogs produced by the fish-specific genome duplication severely limits attempts to detect structured RNAs by comparing paralogous regions of the same genome. We have tested this in a preliminary study using paralogous sequences from the fugu genome as input for RNAz. With this ansatz, we recovered only 283 of the 454 fugu ncRNAs known at the time (Ensembl-45). Despite the inherent limitations of "comparative genomics with a single genome" due to massive loss of duplicated genes, we feel that further methodological improvements are worthwhile; these will focus in particular on increasing the sensitivity of detecting paralogous regions.

\section{Discussion}

We have reported here on an unbiased survey for evolutionary conserved structured ncRNAs in the currently available genomes of teleosts. As in other metazoan animals, we find evidence for several thousand structured RNA motifs of which only a small fraction can be annotated. Due to the large evolutionary distances among teleosts compared to mammals, our RNAz screen has a decreased sensitivity. The absolute value of 11543 structured elements thus cannot be fairly compared with the much larger number of predictions for mammalian genomes ${ }^{8,9}$.

Furthermore, the overwhelming majority of the signals is specific to teleosts, i.e., almost no homologous sequences can be identified in invertebrates. Nevertheless, we have identified several hundred previously unannotated candidates that are shared between teleosts and tetrapods. Conversely, the majority of those RNAz hits that have homologous sequences in other vertebrates can still be recognized as structured 

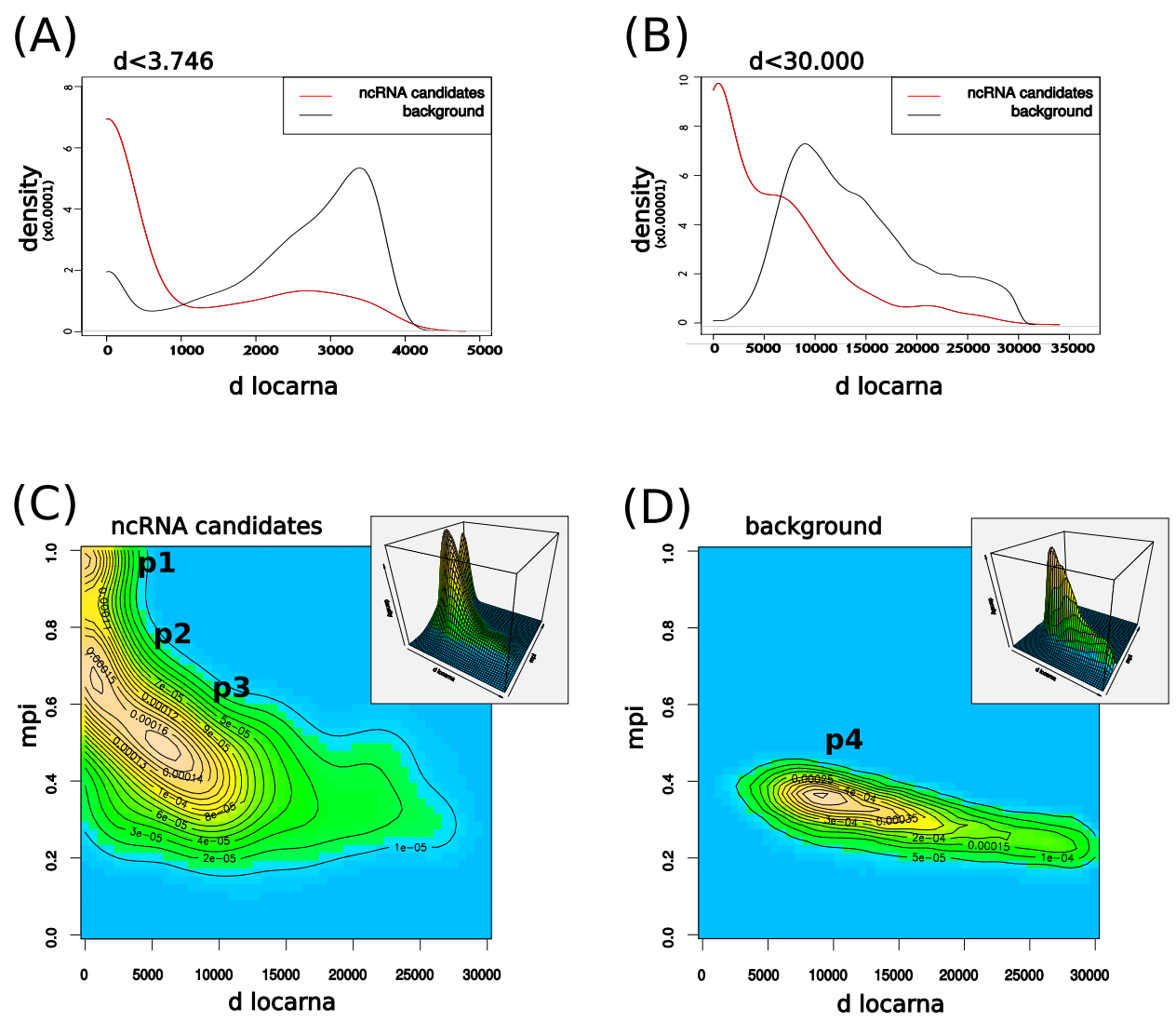

Fig. 7. Distribution of structure distances for duplicated and all ncRNA candidates.

The figure illustrates the density plot of the distribution of all pairwise LocARNA distances of putatively duplicated pairs with recognizable sequence similarity (red curve) and the background distribution of randomly selected pairs (black curve) for all reasonable LocARNA alignments, i.e. positive alignment scores (A), and the complete distribution excluding 25 outliers (B). Subsequent to duplication events, the majority of multi-copy genes preserves its secondary structure, however, a substantial fraction of genes displays highly diverged secondary structures, comparable to the distance of random pairs. (C): The bivariate density of mean pairwise identity versus LocARNA distance of pairs of duplicated ncRNA candidates displays three distinct peaks, corresponding to genes which are highly similar at both sequence and secondary structure level (p1), genes with high structural similarity and diverged sequences (p2), and genes which show a degree of divergence at sequence and structure level (p3), comparable to the background distribution of random pairs (p4), see (D). In contrast to (A), (B), and (C), where all possible pairs have been considered as background distribution, (D) includes only 150000 randomly chosen pairs to reduce the computation time for ClustalW alignments.

RNAs at the expanded phylogenetic range. There is strong evidence for the existence of previously undescribed structurally defined ncRNA families from structure-based clustering, see Fig. 5.

Following the FSGD, we observe that very few ncRNAs retain recognizable 
duplicates. Indeed, only a small minority of structured ncRNAs appears in a copy number between 2 and 8 . The overwhelming majority are single copy loci, while at the same time about $12 \%$ of the loci are accounted for by multi-copy gene families (note that the latter number is an underestimate, since it excludes for instance tRNA genes). It appears, therefore, that with the exception of a few RNA classes, most notably microRNAs, large-scale duplication events do not lead to a corresponding increase in the ncRNA repertoire - at least as far as RNAs are concerned that depend on a well-defined structure. One immediate implication is that comparative approaches within the same genome, i.e., comparisons between paralogous regions, will have very limited sensitivity for ncRNA discovery.

\section{Supplement}

Machine readable sequence and annotation files of the Takifugu rubripes RNAz predictions, supplemental text, figures and tables are available at http://www.bioinf . uni-leipzig.de/Publications/SUPPLEMENTS/07-024/

\section{Acknowledgments}

This work has been funded, in part, by the German DFG under the auspieces of the Bioinformatics Initiative BIZ-6/1-2 and the SPP1174 "Deep Metazoan Phylogeny" and by the 6th Framework Programme of the European Union (SYNLET). Qiang LI's visit to Leipzig in fall 2006 was supported in part by the "Vereinigung von Förderern und Freunden der Universität Leipzig e.V." and by grants to Prof. Bailin HAO (Fudan University, Shanghai, China). We are grateful to Tanja Gesell and Stefan Washietl for their offer to use SISSIz before it was published.

\section{References}

1. The ENCODE Project Consortium, Identification and analysis of functional elements in $1 \%$ of the human genome by the ENCODE pilot project, Nature 447 (2007) 799816.

2. P. Carninci, Constructing the landscape of the mammalian transcriptome., J Exp Biol 210 (2007) 1497-1506.

3. M. Pheasant, J. S. Mattick, Raising the estimate of functional human sequences, Genome Res 17 (2007) 1245-1253.

4. P. Kapranov, J. Cheng, S. Dike, D. Nix, R. Duttagupta, A. T. Willingham, P. F. Stadler, J. Hertel, J. Hackermüller, I. L. Hofacker, I. Bell, E. Cheung, J. Drenkow, E. Dumais, S. Patel, G. Helt, G. Madhavan, A. Piccolboni, V. Sementchenko, H. Tammana, T. R. Gingeras, RNA maps reveal new RNA classes and a possible function for pervasive transcription, Science 316 (2007) 1484-1488.

5. N. C. Lau, E. C. Lai, Diverse roles for RNA in gene regulation, Genome Biol 6 (2005) 315.

6. J. S. Mattick, I. V. Makunin, Non-coding RNAs, Hum Mol Genet 15 (2006) R17-29.

7. A. F. Bompfünewerer, C. Flamm, C. Fried, G. Fritzsch, I. L. Hofacker, J. Lehmann, K. Missal, A. Mosig, B. Müller, S. J. Prohaska, B. M. R. Stadler, P. F. Stadler, 
A. Tanzer, S. Washietl, C. Witwer, Evolutionary patterns of non-coding rnas, Th Biosci 123 (2005) 301-369.

8. S. Washietl, I. L. Hofacker, M. Lukasser, A. Hüttenhofer, P. F. Stadler, Mapping of conserved RNA secondary structures predicts thousands of functional noncoding RNAs in the human genome, Nat Biotechnol 23 (2005) 1383-1390.

9. S. Washietl, J. S. Pedersen, J. O. Korbel, C. Stocsits, A. R. Gruber, J. H. et al., Structured RNAs in the ENCODE selected regions of the human genome, Genome Res 17 (2007) 852-864.

10. E. Torarinsson, M. Sawera, J. Havgaard, M. Fredholm, J. Gorodkin, Thousands of corresponding human and mouse genomic regions unalignable in primary sequece contain common RNA structure, Genome Res 16 (2006) 885-889.

11. A. Meyer, Y. Van de Peer, From 2R to 3R: evidence for a fish-specific genome duplication (FSGD), BioEssays 27 (2005) 937-945.

12. K. D. Crow, P. F. Stadler, V. J. Lynch, C. T. Amemiya, G. P. Wagner, The fish specific Hox cluster duplication is coincident with the origin of teleosts, Mol Biol Evol 23 (2006) 121-136.

13. K. Missal, D. Rose, P. F. Stadler, Non-coding RNAs in Ciona intestinalis, Bioinformatics 21 Suppl 2 (2005) ii77-ii78.

14. K. Missal, X. Zhu, D. Rose, W. Deng, G. Skogerbo, R. Chen, P. F. Stadler, Prediction of structured non-coding RNAs in the genomes of the nematodes Caenorhabditis elegans and Caenorhabditis briggsae, J Exp Zoolog B Mol Dev Evol 306B (2006) 379-392.

15. D. Rose, J. Hackermüller, S. Washietl, S. Findeiß, K. Reiche, J. Hertel, P. F. Stadler, S. J. Prohaska, Computational RNomics of drosophilids, BMC Genomics 8 (2007) 406.

16. S. Steigele, W. Huber, C. Stocsits, P. F. Stadler, K. Nieselt, Comparative analysis of structured RNAs in S. cerevisiae indicates a multitude of different functions, BMC Biology 5 (2007) 25.

17. D. Rose, J. Hertel, K. Reiche, P. F. Stadler, J. Hackermüller, NcDNAlign: Plausible multiple alignments of non-protein-coding genomic sequences, Genomics, in press.

18. S. Washietl, I. L. Hofacker, P. F. Stadler, Fast and reliable prediction of noncoding RNAs, Proc Natl Acad Sci USA 102 (2005) 2454-2459.

19. S. Washietl, I. L. Hofacker, Consensus folding of aligned sequences as a new measure for the detection of functional RNAs by comparative genomics, J Mol Biol 342 (2004) $19-30$.

20. T. Babak, B. J. Blencowe, T. R. Hughes, Considerations in the identification of functional RNA structural elements in genomic alignments, BMC Bioinformatics 8 (2007) 33.

21. T. Gesell, S. Washietl, Dinucleotide controlled null models for comparative RNA gene prediction., BMC Bioinformatics 9 (2008) 248.

22. L. Kong, Y. Zhang, Z. Ye, X. Liu, S. Zhao, L. Wei, G. Gao, CPC: assess the proteincoding potential of transcripts using sequence features and support vector machine, Nucleic Acids Res 35 (2007) W345-W349.

23. The Athanasius F. Bompfünewerer Consortium, RNAs everywhere: genome-wide annotation of structured RNAs, J Exp Zoolog B Mol Dev Evol 308 (2007) 1-25.

24. S. Griffiths-Jones, S. Moxon, M. Marshall, A. Khanna, S. R. Eddy, A. Bateman, Rfam: annotating non-coding RNAs in complete genomes, Nucleic Acids Res 33 (2005) D121D124.

25. C. Liu, B. Bai, G. Skogerbo, L. Cai, W. Deng, Y. Zhang, D. Bu, Y. Zhao, R. Chen, NONCODE: an integrated knowledge database of non-coding RNAs, Nucleic Acids 
Res 33 (2005) D112-D115.

26. S. Griffiths-Jones, miRBase: the microRNA sequence database, Methods Mol Biol 342 (2006) 129-138.

27. P. W. C. Hsu, H. Huang, S. Hsu, L. Lin, A. Tsou, C. Tseng, P. F. Stadler, S. Washietl, I. L. Hofacker, miRNAMap: genomic maps of microRNA genes and their target genes in mammalian genomes, Nucleic Acids Res 34 (2006) D135-D139.

28. M. Szymanski, V. A. Erdmann, J. Barciszewski, Noncoding RNAs database (ncRNAdb), Nucleic Acids Res 35 (2007) D162-D164.

29. T. M. Lowe, S. R. Eddy, tRNAscan-SE: A program for improved detection of transfer RNA genes in genomic sequence, Nucleic Acids Res 25 (1997) 955-964.

30. J. Hertel, I. L. Hofacker, P. F. Stadler, snoReport: Computational identification of snoRNAs with unknown targets, Bioinformatics 24 (2008) 158-164.

31. J. Hertel, P. F. Stadler, Hairpins in a haystack: Recognizing microRNA precursors in comparative genomics data, Bioinformatics 22 (2006) e197-e202, ISMB 2006 contribution.

32. S. Will, K. Reiche, I. L. Hofacker, P. F. Stadler, R. Backofen, Inferring noncoding RNA families and classes by means of genome-scale structure-based clustering, PLoS Comput Biol 3 (2007) e65.

33. W. Ritchie, M. Legendre, D. Gautheret, RNA stem-loops: to be or not to be cleaved by RNAse III, RNA 13 (2007) 457-462.

34. Y. Altuvia, P. Landgraf, G. Lithwick, N. Elefant, S. Pfeffer, A. Aravin, M. J. Brownstein, T. Tuschl, H. Margalit, Clustering and conservation patterns of human microRNAs, Nucleic Acids Res 33 (2005) 2697-2706.

35. C. Quezada, C. Navarro, R. San Martin, M. Alvarez, A. Molina, M. I. Vera, Genomic organization of nucleolin gene in carp fish: evidence for several genes, Biol Res 39 (2006) 353-365.

36. A. Mosig, M. Guofeng, B. M. R. Stadler, P. F. Stadler, Evolution of the vertebrate Y RNA cluster, Th Biosci 126 (2007) 9-14.

37. J. Perreault, J. P. Perreault, G. Boire, Ro-associated Y RNAs in metazoans: Evolution and diversification, Mol Biol Evol 24 (2007) 1678-1689.

38. A. Woolfe, M. Goodson, D. K. Goode, P. Snell, G. K. McEwen, T. Vavouri, et al., Highly conserved non-coding sequences are associated with vertebrate development, PLoS Biol 3 (2005) e7.

39. J. Hertel, M. Lindemeyer, K. Missal, C. Fried, A. Tanzer, C. Flamm, I. L. Hofacker, P. F. Stadler, The Students of Bioinformatics Computer Labs 2004 and 2005, The expansion of the metazoan microRNA repertoire, BMC Genomics 7 (2006) 15.

40. O. Jaillon, J. Aury, F. Brunet, J. Petit, N. Stange-Thomann, E. Mauceli, et al., Genome duplication in the teleost fish Tetraodon nigroviridis reveals the early vertebrate proto-karyotype, Nature 431 (2004) 946-957. 

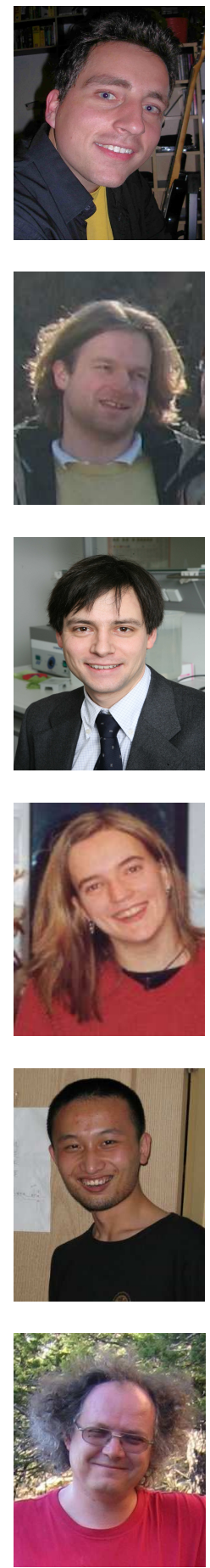

Dominic Rose received his Diploma in Computer Science from U. Leipzig in 2006; he is now working on his $\mathrm{PhD}$ dissertation in Bioinformatics, focussing on ncRNA detection and annotation.

Julian Jöris is studying Computer Science focused on Bioinformatics at U. Leipzig.

Jörg Hackermüller received his $\mathrm{PhD}$ in Chemistry from U. Vienna in 2004. After post doctoral positions at the Novartis Institutes for Biomedical Research, Vienna and at the Fraunhofer Institute for Cell Therapy and Immunology, Leipzig, he is currently leading the RNomics group at Fraunhofer IZI.

Kristin Reiche received her PhD in Computer Science in 2007 focussing on ncRNA detection and annotation. She is now a staff scientist with the RNomics group at Fraunhofer IZI.

Qiang LI is a PhD student focussing on plant ncRNA detection at the T-Life Research Center, working with Prof. Bailin HAO at Fudan University, Shanghai

Peter F. Stadler received his PhD in Chemistry from U. Vienna in 1990 and then worked as Assistant and Associate Professor for Theoretical Chemistry at the same School. In 2002 he moved to Leipzig as Full Professor for Bioinformatics. Since 1994 he is External Professor at the Santa Fe Institute. He helped to found the RNomics group at FH IZI in 2005. 